\title{
The first genome sequence derived from buffalo highly similar to porcine-origin sequences reveals potential cross-species transmission of Seneca Valley virus
}

Xia Zhou ${ }^{1}$, Weifang Liang ${ }^{1}$, Dianhong $\mathrm{Lv}^{2}$, Guangbin $\mathrm{Si}^{3}$, $\mathrm{JH} \mathrm{Li}^{4}$, Zhifei Chen ${ }^{3}$, Weiyou $\mathrm{Cai}^{3}$, Qi Zhai ${ }^{2}$, Shao-Lun Zhai ${ }^{5}$, and Dong-Sheng $\mathrm{He}^{3}$

${ }^{1}$ College of Veterinary Medicine, South China Agricultural University

${ }^{2}$ Institute of Animal Health, Guangdong Academy of Agricultural Sciences

${ }^{3}$ South China Agricultural University

${ }^{4}$ Affiliation not available

${ }^{5}$ Institute of Animal Health

October 29, 2020

\begin{abstract}
Seneca Valley virus (SVV) strain (SVA/GD/China/2018) was first isolated from a buffalo farm in Guangdong, China, using seven mammalian cell lines. A genetic analysis revealed that SVA/GD/China/2018 had a high nucleotide similarity with the porcine-origin SVV strains, revealing potential cross-species transmission of SVV from pigs to buffaloes.
\end{abstract}

The first genome sequence derived from buffalo highly similar to porcine-origin sequences reveals potential cross-species transmission of Seneca Valley virus

Running Title: First report of SVV infection in buffalo

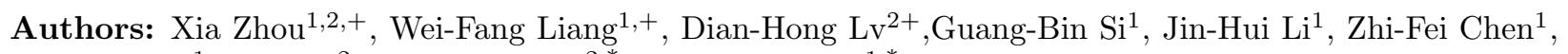
Wei-You Cai ${ }^{1}$, Qi Zhai ${ }^{2}$, Shao-Lun Zhai ${ }^{2, *}$, Dong-Sheng He ${ }^{1, *}$

${ }^{+}$These authors contributed equally to this study.

Affiliations:

College of Veterinary Medicine, South China Agricultural University, Guangzhou, China;

Institute of Animal Health, Guangdong Academy of Agricultural Sciences, Scientific Observation and Experiment Station of Veterinary Drugs and Diagnostic Techniques of Guangdong Province, Ministry of Agriculture of Rural Affairs, and Key Laboratory of Animal Disease Prevention of Guangdong Province, Guangzhou, 510640, China.

Address for correspondence:

Shao-Lun Zhai, Animal Disease Diagnostic Center, Institute of Animal Health, Guangdong Academy of Agricultural Sciences, Guangzhou, 510640, China, zhaishaolun@163.com; Dong-Sheng He, College of Veterinary Medicine, South China Agricultural University, Guangzhou, 510642, China, dhe@scau.edu.cn.

Abstract: Seneca Valley virus (SVV) is an emerging picornavirus, which mainly affects swine health. However, previous studies showed that mice could be infected by SVV. In addition, the antibodies against 
SVV were detected in bovine blood. The data reveal the possible cross-species transmission of SVV. In this study, one SVV strain (SVA/GD/China/2018) was first isolated from a buffalo farm in Guangdong, China, using seven mammalian cell lines. Compared with the 35 reference SVV strains, sequence analysis showed that SVA/GD/China/2018 shared $93.4 \%-99.1 \%$ and $97.5 \%-99.4 \%$ similarity in complete genome level and polyprotein gene level, respectively. Interestingly, SVA/GD/China/2018 shared the highest nucleotide similarity $(99.1 \%$ ) with one wild boar strain (Sichuan HS-01). Genetic analysis revealed that SVA/GD/China/2018 clustered with the porcine-origin SVV strains. Isolation of SVV in buffalo further supported potential cross-species transmission of SVV.

Keywords: Seneca Valley virus; porcine; buffalo; cross-species transmission

\section{1 | Introduction}

Seneca Valley virus (SVV), previously termed Senecavirus A (SVA), is the only member of theSenecavirus genus in the Picornaviridae family. A typical SVV genome structure is L-VP4-VP2-VP3-VP1-2A-2B-2C3A-3B-3C-3D. Both the 5' and 3' ends are untranslated regions (UTRs) (Sangita et al., 2008). Pigs infected with SVV primarily present with vesicular rash on the nose and hoof beetles (Raquel et al., 2016). In severe cases, limp and death occurred due to acute myocarditis, heart degeneration, transient fever, and necrosis (Vannucci et al., 2015). Although SVV could be detected in samples as early as 1988 (Wang, Prarat, Hayes, \& Zhang, 2016), it did not cause any obvious clinical symptoms before 2008. Sporadic outbreaks of obviously pathogenic SVV occurred between 2008 and 2014. Since 2015, large-scale outbreaks have appeared in the United States, Canada, Brazil, China, Italy, Thailand, and Vietnam (Liu et al., 2019; Raquel et al., 2016; Vannucci et al., 2015; Wang, Prarat, Hayes, \& Zhang, 2016). SVV has been detected in mice, houseflies, environmental equipment, corridors in pig farms, and was successfully isolated from mouse tissues (Lork et al., 2016). However, there are no reports of SVV in buffalo. In this study, one SVV strain was first isolated from a buffalo farm in Guangdong, China. The virus was successfully cultured in BHK-21 and NA cells (mouse-origin), PK-15 and ST cells (pig-origin), Vero and Marc-145 cells (monkey-origin) and MDBK cells (bovine-origin). In addition, the viral genome sequence was obtained and characterized.

\section{2 | MATERIALS AND METHODS}

In October 2018, FMD-like clinical presentations, including fever, hoof decay, and a limp, occurred in three buffaloes in a buffalo farm $(\mathrm{n}=80)$ in Guangdong, China. Three oral swab fluids were collected by the farm owner and transported to the Animal Disease Diagnostic Center, Institute of Animal Health, Guangdong Academy of Agricultural Sciences using an insulated container with an ice pack. We extracted the nucleic acids from the oral swab fluids and used reverse transcription PCR to detect FMDV, VSV, BVDV, BTV, and BoHV-1 (Marlise et al., 2005) (Appendix Table) according to the reference protocols and SVV, including detection and genome amplification (Table 1). We used eight cell types from four different origins to isolate and purify the virus. We conducted a genetic evolutionary tree based on the SVV polyprotein with 1000 bootstrap replicates using MEGA6.06 software (Neighbor-Joining). Our results indicated that there was full-length nucleotide and amino acid sequence similarity in the alignment between SVA/GD/China/2018 and other 35 SVV strains published from China and other countries by MegAlign (DNAStar Lasergene.v7.1) software using Clustal W.

\section{3 | RESULTS}

Via RT-PCR test, SVV RNA was positive in those collected samples, and the genome was subsequently amplified. The buffalo-origin SVV was termed, SVA/GD/China/2018 (GenBank Accession No. MN615881). The virus was purified using the double agarose mulching method and cultured it stably in BHK-21 and NA cells, PK-15 and ST cells, Vero and Marc-145, MDBK cells propagated to 30 passages, MDCK cells were only propagated to three passages (Appendix Figure).

The sequence analysis showed that the genome similarity of SVA/GD/China/2018 was $93.4 \%-99.1 \%$, and the polyprotein similarity was $97.5 \%-99.4 \%$ compared with the other 35 SVV strains (Table 2). Interestingly, SVA/GD/China/2018 shared the highest nucleotide similarity with the wild boar strain, Sichuan HS-01 
(99.1\%), and the highest polyprotein similarity with the KS15-01-like strain (99.4\%). Genetic evolutionary analysis revealed that SVA/GD/China/2018 clustered in the same branch with Sichuan HS-01 from Sichuan, China (Figure 1).

Compared with the published SVV sequences, SVA/GD/China/2018 was found to have seven unique amino acid mutations (Figure 2) as follows: ${ }^{440} \mathrm{~A}$ (Alanine) - V (Valine), ${ }^{497} \mathrm{E}$ (Glutamic acid) - K (Lysine), and ${ }^{511} \mathrm{~A}$ (Alanine) - V (Valine) at the VP3 protein; ${ }^{1119} \mathrm{~V}$ (Valine) - I (Isoleucine) at the $2 \mathrm{C}$ protein; ${ }^{1430} \mathrm{~A}$ (Alanine) $-\mathrm{V}$ (Valine) at the $3 \mathrm{~A}$ protein; ${ }^{1710} \mathrm{H}$ (Histidine) $-\mathrm{Y}$ (Tyrosine) at the $3 \mathrm{C}$ protein; and ${ }^{1854} \mathrm{~V}$ (Valine) - I (Isoleucine) at the 3D protein.

\section{4 | DISCUSSION}

PER.C6 is a human cell line, SVV was originally used as a safe and effective oncolytic agent. It has previously been reported that SVV can be isolated from mice in pig farms and can be used as an animal model for SVV disease research. Thus, BHK-21 cells can be used for SVV research since it is a cell line of mouse origin (Liu et al., 2019; Lork et al., 2016). Both PK-15 and ST cells are porcine-origin cell lines, and most SVV strains have been isolated from pigs (Liu et al., 2019; Lork et al., 2016; Raquel et al., 2016; Vannucci et al., 2015; Wang, Prarat, Hayes, \& Zhang, 2016). Virus isolation in this study was established using mouse-origin cell lines (BHK-21 and NA cells), porcine-origin cell lines (PK-15 and ST cells), monkey-origin cell lines (Vero and Marc-145 cells), bovine-origin cell line (MDBK cells), and canine-origin cell line (MDCK cells). It was found that with the exception of MDCK cells, SVV could be continuously stably passaged in all these cell lines from different species, which suggest SVV had potential of cross-species transmission. Moreover, SVV transmission was reported to be nearly $70 \%$ in China in Guangdong (Hause, Myers, Duff, \& Hesse, 2016). SVV can infect pigs of different ages (Nestor et al., 2016), as well as mice; however, this was the first time that SVV was detected in the oral swab fluid of infected buffalo exhibiting clinical symptoms. Moreover, SVV antibodies could be detected in the sera of pigs, mice, and cattle (Yang, Rebekah., 2012). This study confirms the perspective in previous reports that SVV antibodies were detected in bovine serum, buffalo may also be a host of SVV (Lork et al., 2016). In addition, it was established that the buffalo on the farm were both captive and free-range. There were at least five small pig farms within a radius of $3 \mathrm{~km}$ around the buffalo farm, from which nearly 600 pigs were infected. This shows that the host range of the virus is expanding; thus, the media and means of transmission were diversified (Lork et al., 2016).

The genetic evolutionary tree indicated that the SVA/GD/China/2018 strain has a close relationship with the wild boar strain, Sichuan HS-01. Coincidentally, the host of these two strains is not the domestic pig and was also isolated in 2018, whereas the wild boar strain, Sichuan HS-01, was isolated in May, and the SVA/GD/China/2018 strain occurred in October. It is important to note that the SVA/GD/China/2018 and the SVV strains from pigs around the cattle farm share the highest nucleotide and higher amino acid similarity and were located on the same branch with the wild boar strain, Sichuan HS-01. From a timing perspective, the buffalo infection may be related to the infection of the surrounding pigs; however, this strain and the wild boar strain in Sichuan may have evolved from the Guangdong domestic pig strain. These findings demonstrate that there was a relatively close nucleotide relationship with SVV from non-commercial pigs. The strain shared the highest identity with the KS15-01-like strain, indicating that the strain was still new (Wang et al., 2019).

Interestingly, SVA/GD/China/2018 shared the same mutual amino acids at positions, ${ }^{427} \mathrm{~K}$ (Lysine, VP2 protein) and ${ }^{2104} \mathrm{R}$ (Arginine, 3D protein), as the first wild boar strain, Sichuan HS-01 (GenBank Accession No. MH588717), in China. In contrast, the other commercial pig strains had only one or two mutations. This finding reveals that the virus may have undergone adaptive changes in different hosts (Xu et al., 2017); however, further research is required to elucidate the role of these two sites. VP1 contains a hypervariable region with at least two antigenic sites located at the amino acid 140-160 and 200-213 sites (Saeng-Chuto, Rodtian, Temeeyasen, Wegner, \& Nilubol, 2018). It has been reported that ${ }^{228} \mathrm{~K}$ in VP1, ${ }^{141-143} \mathrm{LDV}$, and ${ }^{143-148}$ DGK in VP2 are the primary antigenic sites of FMDV (Bai et al., 2014). None of these three motif sites and antigenic sites of the SVA/GD/China/2018 strain have changed, indicating similar antigenicity and biological characteristics of this strain compared to others (Chen et al., 2017). The majority of the 
characteristics are unique, as the mutations were located in the VP3 protein (3/7); however, further research is required to support the effect of these changes.

In summary, we first isolated a buffalo-origin SVV strain using many of different cell lines. Genetic evolution revealed the possibility of cross-species transmission of SVV.

\section{Acknowledgements}

This study was primarily supported by a grant (Grant No. 2019B020217002) from the Guangdong Provincial Department of Science and Technology, and two grants (Disciplinary Team Construction Program, Grant No. 201634TD and Jinying's Star Talent Program, Grant No. R2018PY-JX003) from Guangdong Academy of Agricultural Sciences, and two grants (Grant Nos. 2019KJ114 and 2019KJ119) from Guangdong Provincial Department of Agriculture and Rural Affairs. Moreover, this study was also partially supported by Maoming Branch and Zhaoqing Branch, Guangdong Laboratory for Lingnan Modern Agriculture, and Scientific Observation and Experiment Station of Veterinary Drugs and Diagnostic Techniques of Guangdong Province, Ministry of Agriculture of Rural Affairs, and the Key Laboratory of Animal Disease Prevention of Guangdong Province.

\section{Disclaimers}

The authors declare no conflict of interest.

\section{Date availability statement}

The date that support the findings of this study are openly available in this manuscript.

\section{References}

Bai, X. W., Bao, H. F., Li, P. H., Wei, W., Zhang, M., Sun, P., .. Liu, Z. (2014). Effects of two amino acid substitutions in the capsid proteins on the interaction of two cell-adapted PanAsia-1 strains of footand-mouth disease virus serotype $\mathrm{O}$ with heparan sulfate receptor. Virology Journal, 11, 132.

Chen, Y., Xu, Q. M., Tan, C., Li, X. X., Chi, X. J., Cai, B. X., ... Chen, J. -L. (2017). Genomic analysis of codon usage shows influence of mutation pressure, natural selection, and host features on Senecavirus A evolution. Microbial Pathogenesis, 112, 313-319.

Hause, B. M., Myers, O., Duff, J., \& Hesse, R. A. (2016). Senecavirus A in Pigs, United States, 2015. Emerging Infectious Diseases, 22(7), 1323-1325.

Liu, J. X., Zha, Y. F., Li, H. Z., Sun, Y. W., Wang, F. G., Lu, R., \& Ning, Z. (2019). Novel recombinant Seneca valley virus isolated from slaughtered pigs in Guangdong Province. Virologica Sinica, 34, 722-724.

Lork, R. J., Kristin, A. M., Travis, C., Kyle, S. H., Bryan, M., Joseph, Y., ... Diel, D. G. (2016). Detection of the emerging picornavirus Senecavirus A in pigs, mice, and houseflies. Journal of Clinical Microbiology, 54(6), 1536-1545.

Marlise, P. C., Alice, F. A., Aurea, V. F., Sheila, R. W., Kerlei, C. M., \& Amauri, A. A. (2005). Rapid detection and differentiation of bovine herpesvirus 1 and 5 glycoprotein $\mathrm{C}$ gene in clinical specimens by multiplex-PCR. Journal of Virological Methods, 128(1-2), 183-188.

Nestor, M., Alexandra, B., Guo, B. Q., Vikas, K., Albert, V. G., Hoang, H., .. Lager, K. (2016). Vesicular disease in 9-week-old pigs experimentally infected with Senecavirus A. Emerging Infectious Diseases, 22(7), $1246-1248$.

Raquel, A. L., Thalita, E. S., Brígida, K. A., Selwyn, A. H., Alice, F. A., Yang, M., \& Alfieri, A. A. (2016). Clinical manifestations of Senecavirus A infection in neonatal pigs, Brazil, 2015. Emerging Infectious Diseases, 22(7), 1238-1241.

Saeng-chuto, K., Rodtian, P., Temeeyasen, G., Wegner, M., \& Nilubol, D. (2018). The first detection of Senecavirus A in pigs in Thailand, 2016. Transboundary and Emerging Diseases, 65(1), 285-288. 
Sangita, V., Seshidhar, P. R., Jackie, L., Neeraja, I., Paul, L. H., \& Vijay, S. R. (2008). Structure of Seneca valley virus-001: an oncolytic picornavirus representing a new genus. Structure, NIH Public Access. 16(10), $1555-1561$.

Vannucci, F. A., Linhares, D. C. L., Barcellos, D. E. S. N., Lam, H. C., Collins, J., \& Marthaler, D. (2015). Identification and complete genome of seneca valley virus in vesicular fluid and sera of pigs affected with idiopathic vesicular disease, Brazil. Transbound Emerging Diseases, 62(6), 589-593.

Wang, L. Y., Prarat, M., Hayes, J., \& Zhang, Y. (2016). Detection and genomic characterization of Senecavirus A, Ohio, USA, 2015. Emerging Infectious Diseases, 22(7), 1321-3.

Wang, M. M., Chen, L. L., Pan, S. N., Mou, C. X., Shi, K. C., \& Chen, Z. H. (2019). Molecular evolution and characterization of novel Seneca Valley virus (SVV) strains in South China. Infection Genetics and Evolution, 69, 1-7.

Xu, W. H., Kate, H., Melissa, G., Bradley, P., Tim, S., Lung, O., \& Nfon, C. (2017). Genome wide analysis of the evolution of Senecavirus A from swine clinical material and assembly yard environmental samples. PLoS One, 12(5), e176964.

Yang, M., Rebekah, V. B., \& Xu, W. H. (2012). Generation and diagnostic application of monoclonal antibodies against Seneca Valley virus. Journal of Veterinary Diagnostic Investigation, 24(1), 42-50.

Table 1 Details of the primers used for the detection and genome amplification of SVV in this study.

\begin{tabular}{|c|c|c|c|}
\hline$\overline{\text { Primers }}$ & Nucleotide sequences (5'-3') & Annealing temperature & Product length (bp) \\
\hline$\overline{\mathrm{SVV}-J C F}$ & ATGGTTGGTTTAGCCTGCACAAG & $59^{\circ} \mathrm{C}$ & 719 \\
\hline SVV-JCR & AAGCACGGATGAGACAGAGTTCCAA & & \\
\hline SVV 1-F & TTTGAAATGGGGGGCTGGGC & $62^{\circ} \mathrm{C}$ & 482 \\
\hline SVV 1-R & GTACTCATGGTGGTAGCAGTCACGTGG & & \\
\hline SVV 2-F & ATCACTGAACTGGAGCTCGA & $57^{\circ} \mathrm{C}$ & 1443 \\
\hline SVV 2-R & AGGAGTTCTGTGTCTCTGAGGA & & \\
\hline SVV 3-F & AGTCTCTTGGCACATACTATCGG & $58^{\circ} \mathrm{C}$ & 1614 \\
\hline SVA 3-R & AAGCACGGATGAGACAGAGTTC & & \\
\hline SVV 4-F & TTAAGGTACTGGAGAAGGACGC & $57^{\circ} \mathrm{C}$ & 1385 \\
\hline SVV 4-R & TGGCATTGATCATAGTGGTGAG & & \\
\hline SVV 5-F & TTGGCTCATGATGCCTTCAT & $56^{\circ} \mathrm{C}$ & 1437 \\
\hline SVV 5-R & GTCCAAACTTGTCTAGATTGTTAGGG & & \\
\hline SVV 6-F & CAACAGACCTTCTGGACTTACAC & $57^{\circ} \mathrm{C}$ & 1505 \\
\hline SVV 6-R & AGAGCAGTCCTGATGATCACA & & \\
\hline SVV 7-F & CTCCTTCGAGGCTCTCATCT & $58^{\circ} \mathrm{C}$ & 707 \\
\hline SVV 7-R & TCTGTTCCGACTGAGTTCTCC & & \\
\hline
\end{tabular}

Table 2 Partial nucleotide and amino acid percentage identities of the SVA/GD/China/2018 strain compared with other SVV strains.

\begin{tabular}{llllll}
\hline Country & Area & Strain & GenBank Number & Nucleotide Similarity & Polyprotein Sin \\
\hline China & Guangdong & CH-GDYD-2017 & MG428683 & $97.3 \%$ & $98.8 \%$ \\
& Guangdong & CH-GDLZ02-2017 & MG428681 & $97.4 \%$ & $98.8 \%$ \\
& Guangdong & GD06/2017 & MH316117 & $97.6 \%$ & $99.1 \%$ \\
& Guangdong & CH-01-2015 & KT321458 & $96 \%$ & $98.8 \%$ \\
& Guangxi & SVA/GX/CH/2018 & MK039162 & $96.9 \%$ & $98.6 \%$ \\
& Hei Longjiang & SVA/HLJ/CHA/2016 & KY419132 & $97.9 \%$ & $99.1 \%$
\end{tabular}




\begin{tabular}{llllll}
\hline Country & Area & Strain & GenBank Number & Nucleotide Similarity & Polyprotein Sin \\
\hline \multirow{6}{*}{ Sichuan } & Sichuan HS-01 & MH588717 & $99.1 \%^{*}$ & $99.3 \%$ \\
& Sichuan & SVV-SC-01 & MH716015 & $96.4 \%$ & $98.5 \%$ \\
& Fujian & CH-FJ-2017 & KY747510 & $98.6 \%$ & $99.1 \%$ \\
& Fujian & SVA CH/FuJ/2017 & MH490944 & $97.6 \%$ & $99.0 \%$ \\
& Henan & CH-HNSL-2017 & KY747512 & $98.6 \%$ & $99.0 \%$ \\
& Henan & HN01-2017 & MH064433 & $97.5 \%$ & $98.8 \%$ \\
& Anhui & AH01-CH-2016 & MF460448 & $97.8 \%$ & $99.3 \%$ \\
& Hebei & HB01-2017 & MF967574 & $97.6 \%$ & $98.9 \%$ \\
& Hebei & HB-CH-2016 & KX377924 & $96 \%$ & $98.7 \%$ \\
USA & Kansas & KS15-01 & KX019804 & $98.5 \%$ & $99.4 \%^{*}$ \\
& Iowa & USA/IA44662/2015 P1 & KU954089 & $98.4 \%$ & $99.4 \%^{*}$ \\
\hline
\end{tabular}

* stands for maximum

The SVA/GD/China/2018 was derived from Guangdong and shared the highest nucleotide similarity with the wild boar strain, Sichuan HS-01 (99.1\%), and the highest polyprotein similarity with the KS15-01-like strain (99.4\%).

Figure 1 The phylogenetic analysis of SVV based on polyproteins. Neighbor-joining tree generated with 1000 bootstrap samplings (MEGA 6.06). The diamond shape represents the strains in this study. The icon before the accession number indicates the host source of the sequence. At present, only SVA/GD/China/2018 has been derived from diseased buffalo and is in the same branch and closely related to the wild boar-origin strain, Sichuan HS-01.

Figure 2 Amino acid sequence alignment of the polyproteins.

Appendix Table. Information used for detection in this study

\begin{tabular}{ll}
\hline Virus & Reference protocol \\
\hline FMDV & Shenzhen Aodong Inspection and Testing Technology Co., Ltd. \\
VSV & Guangzhou Vipotion Biotechnology Co., Ltd. \\
BVDV & reference article (Marlise et al., 2005) \\
BTV & reference article (Marlise et al., 2005) \\
BoHV-1 & reference article (Marlise et al., 2005) \\
\hline
\end{tabular}

Appendix Figure. The virus was cultured in eight different cell types (BHK-21, NA, PK-15 ST, Vero, Marc-145, MDBK, MDCK). Obvious cytopathic effects, including cellular rounding, refraction, cell death, shedding, and floating occured in the BHK-21, PK-15, ST, and Vero cells after $36 \mathrm{~h}$. However, only individual cells died and detached in the other four cell types until $96 \mathrm{~h}$ in NA, Marc-145, MDBK, and MDCK cells. 


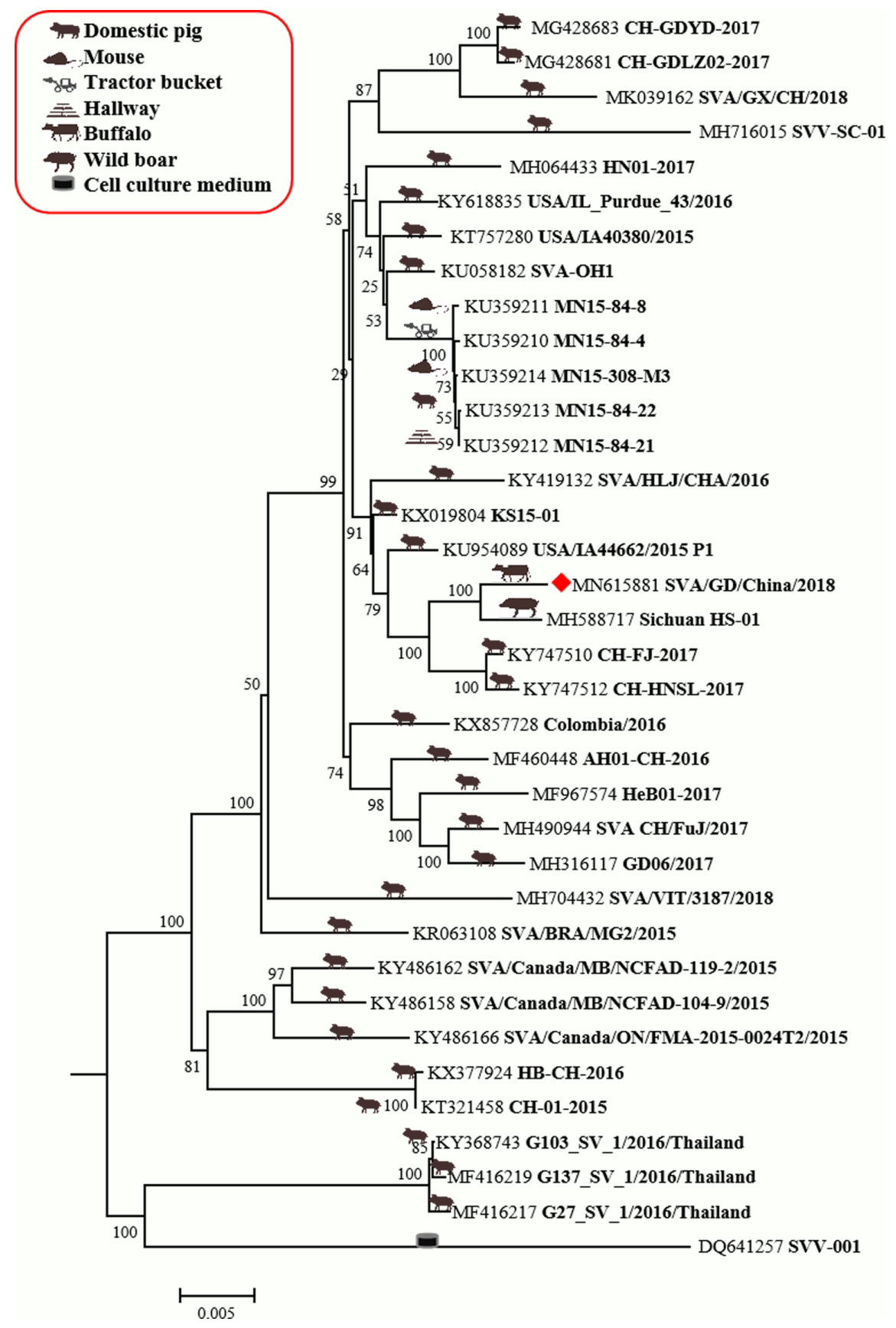




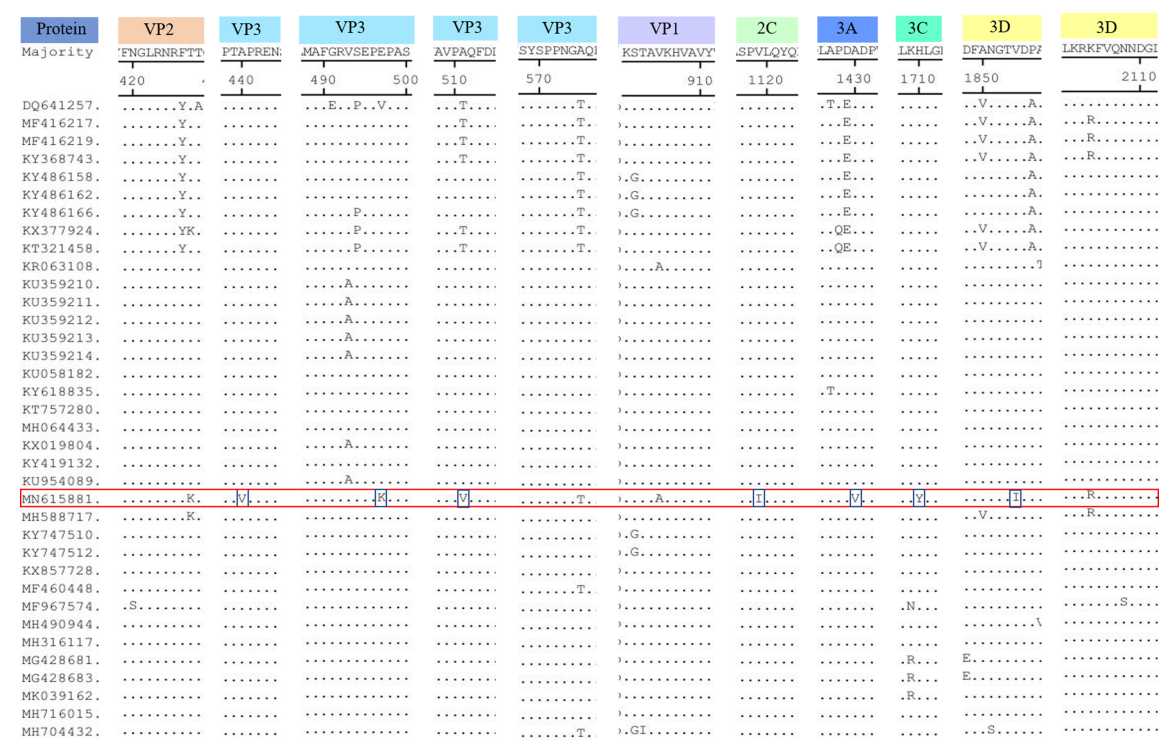

